\title{
Invariant Circles and Rotation Bands In Monotone Twist Maps
}

\author{
Philip L. Boyland* \\ Department of Mathematics, Boston University, Boston, MA 02115, USA
}

\begin{abstract}
In this paper we show that the existence of certain orbits or minimal sets in an area-preserving monotone twist map is necessary and sufficient for the non-existence of invariant circles with specified rotation numbers. The necessity of these conditions follows from classic results of Birkhoff and recent results of Mather. The sufficiency of these conditions depends on the notion of a rotation band which associates a set of rotation numbers with a given orbit or invariant set. We also make some remarks on Mather's paper [M4]. In particular, we use his main theorem to give a lower bound on the width of the interval of rotation numbers associated with the zone of instability that "contains" the irrational $\omega$ when $f$ has no invariant circle with rotation number $\omega$.
\end{abstract}

\section{Section 0}

In this paper we generalize some results of $[\mathrm{B}-\mathrm{H}]$ by showing that the existence of certain orbits or minimal sets in an area-preserving monotone twist map of the annulus is necessary and sufficient for the non-existence of invariant circles with specified rotation numbers. The necessity of these conditions follows from classic results of Birkhoff ([B1, B2 and B3]) and recent results of Mather ([M4]). The sufficiency of these conditions depends on the notion of the rotation band which is defined in Sect. 2. The rotation band of an invariant set is an open interval of real numbers whose endpoints, roughly speaking, quantify the fastest and slowest rate of rotation associated with the set. It can be viewed as a generalization of Birkhoff's definition of an inner and outer rotation number associated with an invariant set that separates the annulus ([B3]).

The main lemma (Lemma 2) states that an area preserving monotone twist maps has no invariant circle whose rotation number lies in the rotation band of an orbit or minimal set. If $f$ preserves the angular order on an invariant set, its rotation band is empty. Thus the non-existence of invariant circles is seen to be

* Current Address: Department of Mathematics, Maharishi International University, Fairfield, IA 52556, USA 
closely related to the existence of orbits on which $f$ does not preserve the angular order. The importance of the order structure of orbits of monotone twist maps was first noted by Aurby and Mather and the notion of an "ordered" invariant set was central to Katok's proof of the Aubry-Mather theorem ([K]).

Our main theorem which is proved in Sect. 2 is:

Main Theorem. If $f: A \rightarrow A$ is an area preserving monotone twist map and $\omega$ is an irrational number between the rotation numbers of $f$ restricted to the boundary circles of $A$, then the following are equivalent:

(1) $f$ has no invariant circle with rotation number $\omega$,

(2) f has a periodic orbit with $\omega$ contained in the rotation band of the periodic orbit,

(3) $f$ has an orbit with $w$ contained in the rotation band of the orbit,

(4) $f$ has a Denjoy minimal set with $\omega$ contained in the rotation band of the minimal set.

A number of other authors have given criterion for the non-existence of invariant circles (see [M-P] for a summary). In most cases, the relationship of these criterion to ours is somewhat unclear.

In the last section of the paper we make some remarks on Mather's paper [M4]. In particular, we use his main theorem in conjunction with our main lemma to give an explicit lower bound on the width of the interval of rotation numbers associated with the zone of instability that "contains" $\omega$ when $f$ has no invariant circle with rotation numbers $\omega$. This bound depends on a number of factors, the most significant being the maximum value of the Peierls' energy barrier at $\omega$ and the Diophantine properties of $\omega$.

\section{Section 1}

We begin by fixing notation. The annulus, $A=S^{1} \times[0,1]$ has universal cover $\tilde{A}=\mathbf{R} \times[0,1]$ with covering projection $p: \tilde{A} \rightarrow A$. The function $f$ will always denote a diffeomorphism $f: A \rightarrow A$ that is isotopic to the identity. The map $F: \tilde{A} \rightarrow \tilde{A}$ is a lift of $f$ if it is a lift in the usual sense and in addition, $F(0,0) \in[0,1] \times\{0\}$. We let $\pi_{1}, \pi_{2}: A \rightarrow \mathbf{R}$ denote the projections onto the first and second coordinates respectively. For each $z \in A$, define the rotation number of $z$ under $f$ as

$$
\rho(z, f)=\limsup _{n \rightarrow \infty} \frac{\pi_{1}\left(F^{n}(\tilde{z})\right)-\pi_{1}(\tilde{z})}{n},
$$

where $p(\tilde{z})=z$. We often suppress the dependence on $f$ and write $\rho(z)$ for $\rho(z, f)$. If $Z$ is an $f$-invariant set, we let $\rho(Z)=\{\rho(z): z \in Z\}$.

An invariant circle for $f$ is an $f$-invariant subset $\Gamma$ which is homeomorphic to $S^{1}$ and is homotopically nontrivial in $A$. Note that if $\Gamma$ is an invariant circle, $f$ restricted to $\Gamma$ is a circle homeomorphism and thus $\rho(\Gamma)$ is a single number. If $f$ has two invariant circles, $\Gamma_{1}$ and $\Gamma_{2}$, and there are no other invariant circles in the annulus bounded by $\Gamma_{1}$ and $\Gamma_{2}$, then this region is called a zone of instability. The map $f$ is an area-preserving monotone twist map if $f$ preserves Lebesgue measure and $\partial \pi_{1} \circ F / \partial y>0$. In addition, to simplify the statements of our theorems we require 
that $f$ can be extended smoothly to a neighborhood of $A$. (This is so we can use Mather's theorem as stated in [M4]). The classic result about invariant circles of such maps is due to Birkhoff ([B1, B2 and B3], for a more modern treatment, with generalizations, see [Fa, H2, K, M1 and M2]).

Theorem. (Birkhoff) If $f: A \rightarrow A$ is an area preserving monotone twist map and $\Gamma$ is an invaraint circle with lift $\tilde{\Gamma}$, then $\tilde{\Gamma}$ is the graph of a Lipschitz function $\mathbf{R} \rightarrow[0,1]$. Further, if $\Gamma_{1}$ and $\Gamma_{2}$ are invariant circles which bound a zone of instability, then given any two neighborhoods $U_{1}$ and $U_{2}$ of $\Gamma_{1}$ and $\Gamma_{2}$, respectively, there exists $z^{\prime}, z \in A$ and integers $n_{1}>n_{2}$ and $n_{1}^{\prime}<n_{2}^{\prime}$ with $f^{n_{1}}(z) \in U_{1}, f^{n_{2}}(z) \in U_{2}, f^{n_{1}^{\prime}}\left(z^{\prime}\right) \in U_{1}$ and $f^{n_{2}}\left(z^{\prime}\right) \in U_{2}$.

We note that there is a uniform Lipschitz bound on the functions whose graphs are the invariant circles of $f([\mathrm{~B} 4, \mathrm{H} 2$ or $\mathrm{K}])$. This implies that the set of invariant circles of $f$ is closed in $A$ and further, that the set, $\{\rho(\Gamma): \Gamma$ is an invariant circle of $f\}$ is closed in $\mathbf{R}$. Thus if $f$ has no invariant circle with $\rho(\Gamma)=\omega$, then there exists a zone of instability with boundary circles $\Gamma_{1}$ and $\Gamma_{2}$ with $\rho\left(\Gamma_{1}\right)<\omega<\rho\left(\Gamma_{2}\right)$.

Next, we introduce the terminology we shall use in our statement of Mather's theorem. As noted in the introduction, invariant sets on which $f$ preserves the angular order have been central to the study of monotone twist maps and have been given a variety of names. We use the definition from [K] and adopt the descriptive terminology from [M-S].

An invariant set $Z$ is called a monotone set under $f$ if

(a) $p^{-1}(Z) \subseteq \tilde{A}$ is the graph of a continuous function $\pi_{1}\left(p^{-1}(Z)\right) \rightarrow[0,1]$,

(b) $F$ restricted to $p^{-1}(Z)$ is order preserving in the first component, i.e.: if $z_{1}, z_{2} \in p^{-1}(Z)$ and $\pi_{1}\left(z_{1}\right)<\pi_{1}\left(z_{2}\right)$, then $\pi_{1}\left(F\left(z_{1}\right)\right)<\pi_{1}\left(F\left(z_{2}\right)\right)$.

Note that if $Z$ is a monotone set, then there exists a homeomorphism $h: S^{1} \rightarrow S^{1}$ with $h\left(\pi_{1}^{\prime}(z)\right)=\pi_{1}^{\prime}(f(z))$ for all $z \in Z$ (here $\pi_{1}^{\prime}: A \rightarrow S^{1}$ is the projection).

Now we extend the notion of a monotone set to that of an $n$-fold monotone set which is an invariant set which lifts to a monotone set in some finite cover. An $n$-fold monotone set looks like the invariant set of a circle homeomorphism that "wraps $n$ times around the annuals." We formalize this notion as follows.

Let $A_{(n)}$ denote the $n$-fold cover of $A$, i.e. $A_{(n)}=\tilde{A} / T^{n}$, where $T: \tilde{A} \rightarrow \tilde{A}$ is $T(x, y)=(x+1, y)$. Let $p_{(n)}: A_{(n)} \rightarrow A$ be the covering projection and $f_{(n)}: A_{(n)} \rightarrow A_{(n)}$ be the lift of $f$ that is the projection of the lift $F: \tilde{A} \rightarrow \tilde{A}$. An $f$-invariant set $Z \subseteq A$ is called an $n$-fold monotone set if there exists an $f_{(n)}$-invariant set $Z_{(n)} \subseteq A_{(n)}$ with $p_{(n)}\left(Z_{(n)}\right)=Z$ and $Z_{(n)}$ is a monotone set under $f_{(n)}$. (For this to make sense, we identify $A_{(n)}$ with the annulus $A$ ) Equivalently, $Z$ is an $n$-fold monotone set if there is an $F$-invariant set $Z^{\prime} \subseteq \tilde{A}$ with $p\left(Z^{\prime}\right)=Z, T^{n}\left(Z^{\prime}\right)=Z$ and further, (a) $Z^{\prime}$ is a graph and (b) $F$ restricted to $Z^{\prime}$ is order preserving (in the sense of (a) and (b) above). Such a lift $Z^{\prime}$ for $Z$ will be called a monotone lift of $Z$. Note that if $j$ divides $n$, any $j$-fold monotone set is also an $n$-fold one.

The $n$-fold monotone sets in Mather's theorem have the additional property of being Denjoy minimal sets. An invariant set $\Sigma \subseteq A$ is a Denjoy minimal set if $(\Sigma, f)$ is topologically conjugate to an exceptional minimal set in a Denjoy circle homeomorphism (see, for example [N] or [M4]). Each Denjoy minimal set carries a unique, invariant probability measure $([\mathrm{Fu}, \mathrm{H} 1])$. These measures may be used 
to put a topology on the set of Denjoy minimal sets for $f$. This topology is called the vague topology (see [M4] for more details). If $\Sigma \subseteq A$ is a Denjoy minimal set that is also an $n$-fold monotone set, we will call $\Sigma$ an $n$-fold Denjoy minimal set. Mather's theorem asserts the existence of many such sets with rotation number $\omega$ for an area preserving monotone twist map with no invariant circle with rotation number $\omega$. It is convenient to parametrize these $n$-fold Denjoy minimal sets by parametrizing their monotone lifts. If $\Sigma$ is an $n$-fold Denjoy minimal set, let $\Sigma^{\prime} \subseteq \tilde{A}$ be a monotone lift. We use the unique invariant probability measure on $\Sigma$ to get an $F$-invariant measure on $\Sigma^{\prime}$, denoted $\mu_{\Sigma^{\prime}}$, with $\mu_{\Sigma^{\prime}}\left(\left\{y \in \Sigma^{\prime}: \xi \leqq \pi_{1}(y) \leqq \xi+n\right\}=n\right.$ for all $\xi \in \mathbf{R}$. We now define $\psi: \Sigma^{\prime} \rightarrow \mathbf{R}$ via $\psi(z)=\mu_{\Sigma^{\prime}}\left(\left\{y \in \Sigma^{\prime}: 0 \leqq \pi_{1}(y) \leqq \pi_{1}(z)\right\}\right)$. Since $\Sigma^{\prime}$ is a monotone lift, we may also define a function $\psi: \mathbf{R} \rightarrow \mathbf{R}$ via $\bar{\psi}(x)=\max \left\{\psi(z): z \in \Sigma^{\prime}\right.$ and $\left.\pi_{1}(z) \leqq x\right\}$. For future reference, we note some properties of $\psi$ and $\bar{\psi}$. These properties can be easily derived from standard results on Denjoy minimal sets using the fact that $\Sigma^{\prime}$ is a monotone lift.

(i) If we let $R_{\omega}: \mathbf{R} \rightarrow \mathbf{R}$ be $R_{\omega}(x)=x+\omega$, then for $z \in \Sigma^{\prime}, \psi \circ F(z)=R_{\omega} \circ \psi(z)$.

(ii) $\psi$ is weakly order preserving, i.e. for $z_{1}, z_{2} \in \Sigma^{\prime}, \pi_{1}\left(z_{1}\right)<\pi_{1}\left(z_{2}\right)$ implies $\psi\left(z_{1}\right) \leqq \psi\left(z_{2}\right)$.

(iii) $\bar{\psi}(x+n)=\bar{\psi}(x)+n$.

(iv) For $b \in \mathbf{R}, \psi^{-1}(\bar{\psi}(b))$ is at most two points.

We can now define the function which will parametrize Mather's $n$-fold Denjoy minimal sets. Given $\xi \in \mathbf{R}$, define $\Psi$ : monotone lifts of $n$-fold Denjoy minimal sets $\} \rightarrow \mathbf{R}^{n}$ by defining its $i^{\text {th }}$ coordinate as,

$$
\Psi_{i}\left(\Sigma^{\prime}\right)=\bar{\psi}(\xi+i)-\bar{\psi}(\xi+i-1) .
$$

Thus $\Psi_{i}\left(\Sigma^{\prime}\right)$ is the measure of the portion $\Sigma^{\prime}$ that lies in the vertical strip $[\xi+i+1, \xi+i] \times[0,1]$. Note that $\Psi$ depends on $\xi$, and we will denote this dependence (when needed) by writing $\Psi_{\xi}$. Also, $\sum_{i=1}^{n} \Psi_{i}\left(\Sigma^{\prime}\right)=\bar{\psi}(\xi+n+1)-$ $\bar{\psi}(\xi+1)=n$.

Mather's theorem states that the image of $\Psi$ contains an $n-1$ dimensional disk. Moreover, he precisely specifies this disk. As this will be crucial to our applications, we give this specification. Given $\delta>0$, let $B_{\delta, n}=\left\{\vec{v} \in \mathbf{R}^{n-1}:\left|v_{i}-v_{j}\right|<\delta\right.$ and $\left|v_{i}\right|<\delta$ for $\left.i, j=1,2, \ldots, n-1\right\}$. Now define an affine map $A: \mathbf{R}^{n-1} \rightarrow \mathbf{R}^{n}$ via $A\left(v_{1}, \ldots, v_{n-1}\right)=\left(1+v_{1}, 1+v_{2}-v_{1}, \ldots, 1+1+v_{n-1}-v_{n-2}, 1-v_{n-1}\right)$ and let $C_{\delta, n}=A\left(B_{\delta, n}\right)$. Since $A$ is injective and $B_{\delta, n}$ is a topological disk, we have that $C_{\delta, n}$ is an $n-1$ dimensional disk. $\Psi$ will turn out to be a homeomorphism onto $C_{\delta, n}$ when the domain of $\Psi$ is restricted to the monotone lifts of a certain collection of $n$-fold Denjoy minimal sets. If $M$ is a collection of $n$-fold Denjoy minimal sets, let $\mathscr{C}(M)=\left\{\Sigma^{\prime} \subseteq \tilde{A}: \Sigma^{\prime}\right.$ is a monotone lift of a $\left.\Sigma \in M\right\}$. The vague topology of $\mathscr{C}(M)$ is obtained from the vague topology on $M$ and the projection $\mathscr{C}(M) \rightarrow M$.

Before stating Mather's theorem, we informally describe its contents. An $n$-fold Denjoy minimal set $\Sigma$, is a minimal set that wraps $n$-times around the annulus. If $\Sigma$ has rotation number $\omega$, the average speed for traversing all $n$ loops is $\omega$. The function $\Psi_{i}$ gives the amount of measure of $\Sigma$ that is contained in the $i^{\text {th }}$ loop. 
Since the invariant measure on $\Sigma^{\prime}$ may be used to define a semiconjugacy to rigid rotation by $\omega$, one may view $\Psi_{i}$ as measuring the "speed" of orbits under iteration in the $i^{\text {th }}$ loop of $\Sigma$. Thus $\Psi$ parametrizes the monotone lift of an $n$-fold Denjoy minimal set via the speeds in each of the $n-1$ loops of its projection to $A$. Since the average speed to traverse all $n$ loops must be $\omega$, there are only $n-1$ free parameters. Mather's theorem asserts the existence of sufficient $n$-fold Denjoy minimal sets so that this parametrization gives "speeds" in an $n$-dimensional ball.

Theorem. (Mather) Let $f: A \rightarrow A$ be an area preserving monotone twist map and $\omega$ be an irrational number between the rotation numbers of $f$ restricted the boundary circles of $A$. If $f$ has no invariant circle with rotation number $\omega$, then there exists a $\xi \in \mathbf{R}$ such that for each positive integer $n$ there exists $a \delta>0$ and a collection $M_{n}$ of $n$-fold Denjoy minimal sets for $f$ so that $\Psi_{\xi}: \mathscr{C}\left(M_{n}\right) \rightarrow C_{\delta, n}$ is a homeomorphism when $\mathscr{C}\left(M_{n}\right)$ is given the vague topology.

The quantities $\xi$ and $\delta$ and the collection $M_{n}$ in this theorem are explicitly described in [M4]. They are given in terms of data derived from the function $f$ and the arithmetic properties of the number $\omega$ and will be described in Sect. 3 .

The implication of this theorem that is perhaps of most interest is obtained by noting that the set $M_{n}$ with the vague topology contains a topological $n-1$ disk. To see this, one projects $\mathscr{C}\left(M_{n}\right) \rightarrow M_{n}$ and notes that the projection is $n$ to 1 except for those exceptional sets in $M_{n}$ which are also $j$-fold Denjoy minimal sets for some $j$ that divides $n$. Mather states his result in this more interesting form. We have chosen the above formulation as it is more suitable for the applications that follow.

\section{Section 2}

In this section we give two preliminary lemmas and then the proof of the main theorem. We begin by associating an open interval of real numbers, called the rotation band, to each $f$-invariant set $Z$. The rotation band of $Z$, denoted $R B(Z)$, will have the property that any invariant circle, $\Gamma$, will satisfy $\rho(\Gamma) \notin R B(Z)$ whenever $Z$ has a dense orbit.

If $Z \subseteq A$ is an $f$-invariant set, define $H_{+}: \mathbf{R} \rightarrow \mathbf{R}$ by $H_{+}(x)=\sup \left\{\pi_{1}(F(z))\right.$ : $\pi_{1}(z) \leqq x$ and $\left.z \in p^{-1}(Z)\right\}$. We note some simple properties of $H_{+}$whose proofs are elementary.

(1) $H_{+}$is weakly order preserving, i.e. if $x_{1}<x_{2}$, then $H_{+}\left(x_{1}\right) \leqq H_{+}\left(x_{2}\right)$.

(2) $H_{+}(x+1)=H_{+}(x)+1$.

(3) For each $x \in \mathbf{R}$, one may define the rotation number of $x, \rho\left(x, H_{+}\right)$, in the obvious way. By virtue of (1) and (2), $\rho\left(x, H_{+}\right)$exists and is independent of the choice of $x$, and so $\rho\left(H_{+}\right)$is a single number.

(4) If there exists a $z \in p^{-1}(Z)$ with $\pi\left(F^{s}(z)\right) \geqq \pi(z)+r$, then $\rho\left(H_{+}\right) \geqq r / s$.

In a certain sense, $\rho\left(H_{+}\right)$captures the fastest rate of rotation associated with $Z$ (although one may have $\rho\left(H_{+}\right)>\rho(z, F)$ for all $z \in Z$ ). One may also define a weakly order preserving map associated with the slowest rate of rotation on $Z$. Define $H_{-}: \mathbf{R} \rightarrow \mathbf{R}$ via $H_{-}(x)=\inf \left\{\pi_{1}(F(z)): \pi_{1}(z) \geqq x\right.$ and $\left.z \in p^{-1}(Z)\right\}$. The rotation band of $Z$ is defined as the open interval $R B(Z)=\left(\rho\left(H_{-}\right), \rho\left(H_{+}\right)\right)$. If $\rho\left(H_{-}\right)=\rho\left(H_{+}\right)$, 
then $R B(Z)$ is the empty set. This will be the case, for example, if $Z$ is a monotone set for $f$. However, if $Z$ is monotone in some $n$-fold cover and not monotone in $A$, it will usually have nontrivial rotation band. In the case where $Z$ is a periodic orbit, Sect. 2 of $[\mathrm{B}-\mathrm{H}]$ gives a technique for computing $R B(Z)$.

Lemma 1. Let $f: A \rightarrow A$ be an area preserving monotone twist map and $\Gamma \subseteq A$ be an invariant circle. If $Z \subseteq A$ is an $f$-invariant set with a dense orbit, then $\rho(\Gamma) \notin R B(Z)$.

Proof. Since clearly $R B(Z) \subseteq R B$ (closure $(Z)$ ), it suffices to prove the result for the case when $Z$ is compact. Since $\Gamma$ is a homotopically nontrivial invariant circle in $A$, it divides the annulus into two invariant subannuli. Since $Z$ has a dense orbit, it must be contained in one of them. We shall assume that $Z$ is contained in the annulus bounded by $\Gamma$ and the inner boundary of $A$ and show that $\rho(\Gamma) \geqq \rho\left(H_{+}\right)$, where $H_{+}$is constructed from $Z$ as above. If $Z$ is contained in the other subannulus, a similar proof shows that $\rho(\Gamma) \leqq \rho\left(H_{-}\right)$. We shall work in the cover, $\tilde{A}$, with $\widetilde{\Gamma}$ and $F$ lifts of $\Gamma$ and $f$, respectively. For the balance of the proof we let $\pi=\pi_{1}$.

First, since $F(z+(1,0))=F(z)+(1,0)$, we have $H_{+}(x)=\sup \{\pi(F(z)): x-1 \leqq$ $\pi(z) \leqq x$ and $\left.z \in p^{-1}(Z)\right\}$. This is the supremum of a continuous function over a compact set and is thus achieved. Therefore, given $x \in \mathbf{R}$, there always exists a $z \in p^{-1}(Z)$ with $\pi(z) \leqq x$ and $\pi(F(z))=H_{+}(x)$.

Next, we prove by induction that if $x \in \mathbf{R}$ and $a \in \Gamma$ with $x \leqq \pi(a)$, then $H_{+}^{n}(x) \leqq \pi\left(F^{n}(a)\right)$ for $n=0,1, \ldots$. This will finish the proof as it clearly implies that $\rho\left(H_{+}\right) \leqq \rho(\Gamma)$. To prove the inductive step using the result of the previous paragraph, find a $z^{\prime} \in p^{-1}(Z)$ with $\pi\left(z^{\prime}\right) \leqq H_{+}^{n}(x)$ and $\pi\left(F\left(z^{\prime}\right)\right)=H_{+}\left(H_{+}^{n}(x)\right)$. Using the inductive hypothesis, $\pi\left(z^{\prime}\right) \leqq H_{+}^{n}(x) \leqq \pi\left(F^{n}(a)\right)$. Now since $z^{\prime}$ is below $\widetilde{\Gamma}$ and to the left of $F^{n}(a)$, the monotone twist hypothesis implies that $\pi\left(F\left(z^{\prime}\right)\right) \leqq \pi\left(F\left(F^{n}(a)\right)\right)$. Thus $H_{+}^{n+1}(x)=\pi\left(F\left(z^{\prime}\right)\right) \leqq \pi\left(F^{n+1}(a)\right)$ as required.

Note that in this proof, the hypothesis that $Z$ has a dense orbit is only used to get $Z$ completely below or above $\Gamma$. Any other conditions on $Z$ which insure this are sufficient to imply the conclusion of the lemma.

If $\Sigma$ is an $n$-fold Denjoy minimal set, the next lemma allows one to compute information about $R B(\Sigma)$ using the function $\psi$ (or equivalently $\Psi$ ) defined in Sect. 1 . The intuitive idea is very simple. If $\rho(\Sigma)=\omega$ and $\Psi\left(\Sigma^{\prime}\right) \neq(1,1, \ldots, 1)$, then orbits are moving with speed faster than $\omega$ in some of the "loops" of the minimal set. This implies that $\rho\left(H_{+}\right)$is bigger than this faster speed. Similar comments hold for $\rho\left(H_{-}\right)$.

In the statement of Lemma 2 we are assuming that the $\xi$ used in the definition of $\Psi$ is equal to zero. This can clearly be done without loss of generality.

Lemma 2. Let $\Sigma \subseteq A$ be an $n$-fold Denjoy minimal set with $\rho(\Sigma)=\omega$ and define $\bar{\psi}: \mathbf{R} \rightarrow \mathbf{R}$ using a monotone lift, $\Sigma^{\prime} \subseteq \tilde{A}$, as in Sect. 1 . If there exist integers $r_{i}, r_{i}^{\prime}, s$ and $s^{\prime}$ for $i=1,2$ which satisfy $\bar{\psi}\left(r_{2}^{\prime}\right)-\bar{\psi}\left(r_{1}^{\prime}\right)>s^{\prime} \omega>r_{2}^{\prime}-r_{1}^{\prime}$ and $\bar{\psi}\left(r_{2}\right)-\bar{\psi}\left(r_{1}\right)<$ $s \omega<r_{2}-r_{1}$, then $\omega \in\left(r_{2}^{\prime}-r_{1}^{\prime}\right) / s^{\prime},\left(r_{2}-r_{1}\right) / s \subseteq R B(\Sigma)$.

Proof. As noted in Sect. 1, for each $b \in \mathbf{R}, \psi^{-1}(\bar{\psi}(b))$ consists of at most two points which we denote as $z_{b}^{\prime}$ and $z_{b}^{\prime \prime}$ and which we assume are chosen so that $\pi_{1}\left(z_{b}^{\prime}\right) \leqq b \leqq \pi_{1}\left(z_{b}^{\prime \prime}\right)$. We claim that the hypotheses of the lemma imply that $\pi_{1}\left(F^{s}\left(z_{r_{1}}^{\prime}\right)\right)>r_{2}$. This will imply that $\rho\left(H_{+}\right) \geqq\left(r_{2}-r_{1}\right) / s$, using property (4) of $H_{+}$ 
given above because

$$
\pi_{1}\left(F^{s}\left(z_{r_{1}}^{\prime}\right)\right)>r_{1}+\left(r_{2}-r_{1}\right) \geqq \pi_{1}\left(z_{r_{1}}^{\prime}\right)+\left(r_{2}-r_{1}\right) .
$$

The proof of the inequality $\rho\left(H_{-}\right) \leqq\left(r_{2}^{\prime}-r_{1}^{\prime}\right) / s^{\prime}$ is similar.

To prove the claim, since $\psi \circ F=R_{\omega} \circ \psi$ on $\Sigma^{\prime}$, then $\psi\left(F^{s}\left(z_{r_{1}}^{\prime}\right)\right)=R_{\omega}^{s}\left(\psi\left(z_{r_{1}}^{\prime}\right)\right)=$ $R_{\omega}^{s}\left(\bar{\psi}\left(r_{1}\right)\right)=\bar{\psi}\left(r_{1}\right)+s \omega>\bar{\psi}\left(r_{2}\right)$. Since $\psi$ is weakly order preserving and $\psi^{-1}\left(\bar{\psi}\left(r_{2}\right)\right)=\left\{z_{r_{2}}^{\prime}, z_{r_{2}}^{\prime \prime}\right\}$, we have $\pi_{1}\left(F^{s}\left(z_{r_{1}}^{\prime}\right)\right)>\pi_{1}\left(z_{r_{2}}^{\prime \prime}\right) \geqq r_{2}$ as required.

We remark that the hypothesis of this lemma necessitates $r_{1} \neq \equiv r_{2} \bmod n$ for if $r_{1}=k n+r_{2}$ for some $k \in \mathbf{Z}$, then $\bar{\psi}\left(r_{1}\right)=\bar{\psi}\left(k n+r_{2}\right)=\bar{\psi}\left(r_{2}\right)+k n$, so $\bar{\psi}\left(r_{1}\right)-\bar{\psi}\left(r_{2}\right)=$ $r_{1}-r_{2}$. A similar argument shows that $r_{1}^{\prime} \neq r_{2}^{\prime} \bmod n$.

Proof of Main Theorem. The equivalence of (1) and (2) is contained in [B-H]. Lemma 1 shows that (3) or (4) imply (1). To see that (1) implies (3), by the remarks following Birkhoff's Theorem, there exists a zone of instability with boundary circles $\Gamma_{1}$ and $\Gamma_{2}$ with $\rho\left(\Gamma_{1}\right)<\omega<\rho\left(\Gamma_{2}\right)$. Now pick rationals $p_{1} / q_{1}$ and $p_{2} / q_{2}$ with $\rho\left(\Gamma_{1}\right)<$ $p_{1} / q_{1}<\omega<p_{2} / q_{2}<\rho\left(\Gamma_{2}\right)$. Using the continuity of $f$, we may choose neighborhoods $U_{1}$ and $U_{2}$ of $\Gamma_{1}$ and $\Gamma_{2}$, respectively, which are small enough to ensure that $\pi_{1}\left(F^{q_{1}}\left(\tilde{y}_{1}\right)\right)<\pi_{1}\left(\tilde{y}_{1}\right)+p_{1}$ and $\pi_{1}\left(F^{q_{2}}\left(\tilde{y}_{2}\right)\right)>\pi_{1}\left(\tilde{y}_{2}\right)+p_{2}$ whenever $p\left(\tilde{y}_{i}\right) \in U_{i}$. Thus using Birkhoff's theorem, we may find a $\tilde{z} \in \tilde{A}$ and integers $n_{1}$ and $n_{2}$ so that $\pi_{1}\left(F^{q_{1}}\left(F^{n_{1}}(\tilde{z})\right)\right)<\pi\left(F^{n_{1}}(\tilde{z})\right)+p_{1}$ and $\pi_{1}\left(F^{q_{2}}\left(F^{n_{2}}(\tilde{z})\right)\right)>\pi\left(F^{n_{2}}(\tilde{z})\right)+p_{2}$. Therefore, using property (4) for $H_{+}$given in Sect. 1. (and its analogue for $H_{-}$) we have $\omega \in\left(p_{1} / q_{1}, p_{2} / q_{2}\right) \subseteq\left(\rho\left(H_{-}\right), \rho\left(H_{+}\right)\right)=R B(o(z))$, where $z=p(\tilde{z})$.

To see that (1) implies (4), using Mather's theorem, choose any integer $n>1$ and any $\Sigma \subseteq M_{n}$ so that $\Psi\left(\Sigma^{\prime}\right) \neq(1,1, \ldots, 1)$. If this is the case (we have let $\xi=0$ ), since by definition, $\Psi_{i}\left(\Sigma^{\prime}\right)=\bar{\psi}(i+1)-\bar{\psi}(i)$ and $\sum_{i=1}^{n} \Psi_{i}\left(\Sigma^{\prime}\right)=n$, there exist $r_{i}, r_{i}^{\prime} \in$ $\{1, \ldots, n\}$ for $i=1,2$ with $r_{2}>r_{1}$ and $r_{2}^{\prime}>r_{1}^{\prime}$ and $\bar{\psi}\left(r_{2}\right)-\bar{\psi}\left(r_{1}\right)<r_{2}-r_{1}$ and $\bar{\psi}\left(r_{2}^{\prime}\right)-\bar{\psi}\left(r_{1}^{\prime}\right)>r_{2}^{\prime}-r_{1}^{\prime}$. Thus $0<\left(\bar{\psi}\left(r_{2}\right)-\bar{\psi}\left(r_{1}\right)\right) / n<\left(r_{2}-r_{1}\right) / n<1$, and since the orbit of zero in $S^{1}$ under rotation by $\omega / n$ is dense, we may find positive integers $s$ and $k$ with $\left(\bar{\psi}\left(r_{2}\right)-\bar{\psi}\left(r_{1}\right)\right) / n<s(\omega / n)-k<\left(r_{2}-r_{1}\right) / n$, and so $\bar{\psi}\left(r_{2}\right)+k n-\psi\left(r_{1}\right)<$ $s \omega<r_{2}+k n-r_{1}$. But since $\bar{\psi}(x+n)=\bar{\psi}(x)+n$, letting $\bar{r}_{2}=r_{2}+k n$, we have $\bar{\psi}\left(\bar{r}_{2}\right)-\bar{\psi}\left(r_{1}\right)<s \omega<\bar{r}_{2}-r_{1}$. Similarly, we may find a $\bar{r}_{2}^{\prime}$ and $s^{\prime}$ with $\bar{\psi}\left(\bar{r}_{2}^{\prime}\right)-$ $\psi\left(r_{1}^{\prime}\right)>s^{\prime} \omega>\bar{r}_{2}^{\prime}-r_{1}^{\prime}$, and so by Lemma $2, \omega \in\left(\left(\bar{r}_{2}^{\prime}-r_{1}^{\prime}\right) / s^{\prime},\left(\bar{r}_{2}-r_{1}\right) / s\right) \subseteq R B(\Sigma)$.

\section{Section 3}

In this section we make some remarks on Mather's paper [M4]. In particular, we use the specification of the $\delta$ in his theorem, in conjunction with Lemma 2, to give a lower bound for the width of the interval of rotation numbers associated with the zone of instability that "contains" $\omega$ when $f$ has no invariant circle with rotation number $\omega$.

We shall restrict consideration to the class of $f: \mathbf{R}^{2} \rightarrow \mathbf{R}^{2}$ considered in [M4], Sect. 2 and call such a map a area-preserving monotone twist map of the infinite cylinder. Given such an $f: \mathbf{R}^{2} \rightarrow \mathbf{R}^{2}$, there always exists a generating function $h: \mathbf{R}^{2} \rightarrow \mathbf{R}$ with $f(x, y)=\left(x^{\prime}, y^{\prime}\right)$ if and only if $y=-h_{1}\left(x, x^{\prime}\right)$ and $y^{\prime}=h_{2}\left(x, x^{\prime}\right)$, where 
$h_{1}$ and $h_{2}$ denote the partial derivatives with respect to $x$ and $x^{\prime}$. For each irrational $\omega$, using $h$, one may define a continuous function, $P_{\omega}: \mathbf{R} \rightarrow \mathbf{R}$, called the Peierls' energy barrier which has the property that $f$ has an invariant circle with rotation number $\omega$ if and only if $P_{\omega}$ is identically zero (see [M3] Sect. 14 also [A-LD-A]). Since $P_{\omega}$ is periodic, we may define $P(\omega, h)=\max \left\{P_{\omega}(\xi): \xi \in \mathbf{R}\right\}$. We note that the quantity $\Delta W_{\omega}$ studied in [M3] is a lower bound for $P(\omega)$ ([M3] Sect. 25). Mather has examples for which $\Delta W_{\omega}<P(\omega)$.

We now give the specification of $\delta$. Given $h$, an irrational $\omega$ and a positive integer $n$, define $C(h, \omega, n)=\sup \left\{\left|h_{1}\left(x, x^{\prime}\right)\right|+\left|h_{2}\left(x, x^{\prime}\right)\right|: n[\omega / n]-1 \leqq x^{\prime}-x \leqq\right.$ $n[\omega / n]+n+1\}$, where $[\omega / n]$ means the greatest integer $\leqq \omega / n$. Now define $N(h, \omega, n)$ to be the least integer $>4 C(h, \omega, n) / P(\omega, h)$, and let $\delta(h, \omega, n)=\min \{\mid i \omega+$ $j \mid: i, j \in \mathbf{Z}$ and $0<i \leqq N(h, \omega, n)\}$. This $\delta(h, \omega, n)$ is the largest value of $\delta$ which is specified in Mather's theorem. It corresponds to a $\xi$ that satisfies $P_{\omega}(\xi)=P(\omega, h)$, i.e. $\xi$ is any value at which the maximum of the energy barrier is achieved.

We also need to recall some elementary facts about continued fractions (see $[\mathrm{H}-\mathrm{W}]$ or $[\mathrm{N}-\mathrm{Z}]$ for details). Using the standard notation, each irrational $\omega>0$ may be expressed in a unique way as a continued fraction, $\omega=\left[a_{0}, a_{1}, \ldots\right]$ for positive integers $a_{i}$. The $j^{t h}$ convergent of $\omega$ is $p_{j} / q_{j}=\left[a_{0}, a_{1}, \ldots, a_{j}\right]$, where $p_{j} / q_{j}$ is in lowest form. If we let $\Delta_{j}=q_{j} \omega-p_{j}$, then the sequences $\left|\Delta_{j}\right|$ and $\left|p_{j} / q_{j}-\omega\right|$ monotonically decrease to zero and alternate signs according to

$$
\Delta_{j}=(-1)^{j}\left|\Delta_{j}\right| \quad \text { and } \quad \frac{p_{j}}{q_{j}}-\omega=(-1)^{j+1}\left|\frac{p_{j}}{q_{j}}-\omega\right| .
$$

In addition, $p_{j} q_{j-1}-p_{j-1} q_{j}=(-1)^{j+1}$ and $p_{j} q_{j-2}-p_{j-2} q_{j}=(-1)^{j}\left(a_{j}\right)$. Note that this implies that $p_{j}$ and $p_{j+1}$ are relatively prime. Finally, if $r$ and $s$ are positive integers with $|s \omega-r|<\left|q_{k} \omega-p_{k}\right|$, then $s \geqq q_{k+1}$ (see [N-Z], Theorem 7.13). Note that this implies that $\delta(h, \omega, n)=\left|\Delta_{k}\right|$, where $k=\max \left\{i \in \mathbf{N}: q_{i} \leqq N(h, \omega, n)\right.$ and $p_{i} / q_{i}$ is a convergent of $\omega\}$. If $a$ and $b$ are two real numbers, let $\langle a, b\rangle$ denote the convex hull of $\{a, b\}$. For two integers $m$ and $n$, the notation $m \nmid n$ means $m$ does not divide $n$.

Theorem 3. Let $f: \mathbf{R}^{2} \rightarrow \mathbf{R}^{2}$ be an area-preserving monotone twist map of the infinite cylinder with generating function $h, \omega$ be an irrational, $n$ be a positive integer and $N(h, \omega, n)$ be defined as above. If $f$ has no invariant circle with rotation number $\omega$ and we let $j(n)=\min \left\{i: p_{i} / q_{i}\right.$ is a convergent of $\omega$ and $\left.q_{i}>N(h, \omega, n)\right\}$, then for any f-invariant circle $\Gamma$,

$$
\rho(\Gamma) \notin\left\langle\frac{p_{j(n)}+p_{j(n)+1}}{q_{j(n)}+q_{j(n)+1}}, \frac{p_{j(n)+1}+p_{j(n)+2}}{q_{j(n)+1}+q_{j(n)+2}}\right\rangle .
$$

Proof. Fix $n$ and let $\delta(h, \omega, n)=\delta$. We first claim that if $r, s, r^{\prime}$ and $s^{\prime}$ are positive integers which satisfy (1) $n \mid r$ and $n \mid r^{\prime}$ and (2) $0<s^{\prime} \omega-r^{\prime}<\delta$ and $0<r-s \omega<\delta$, then $f$ has no invariant circle $\Gamma$ with $\rho(\Gamma) \in\left(r^{\prime} / s^{\prime}, r / s\right)$. To prove this claim we will produce an $n$-fold Denjoy minimal set $\Sigma$ with $\left(r^{\prime} / s^{\prime}, r / s\right) \subseteq R B(\Sigma)$ and use Lemma 1 . Let $p, d, p^{\prime}$ and $d^{\prime}$ be integers with $0<p, d, d^{\prime}<n, 0<p^{\prime} \leqq n$ and $d \equiv r \bmod n, d^{\prime} \equiv r^{\prime}$ $\bmod n$, and $p^{\prime} \equiv d+p \not \equiv d^{\prime} \bmod n$. Now choose $v \in B_{\delta, n}\left(B_{\delta, n}\right.$ was defined in Sect. 1.) whose components satisfy $v_{d}=v_{d^{\prime}}=v_{p}=\delta_{1}$ and $v_{p^{\prime}}=0$, where $\delta_{1}$ is chosen so that 
$s^{\prime} \omega-r^{\prime}<\delta_{1}<\delta$ and $r-s \omega<\delta_{1}<\delta$. By Mather's theorem, we may find a $\Sigma^{\prime} \in M_{n}$ with $\Psi\left(\Sigma^{\prime}\right)=A(\vec{v})$.

Now using the definitions of $\Psi$ and $\bar{\psi}$ given in Sect. 1 , it is easy to see that if $k \neq 0 \bmod n$, then $\bar{\psi}(k)=k+v_{i}$, where $0<i<n$ and $i \equiv k \bmod n$. If $k \equiv 0 \bmod n$, then $\bar{\psi}(k)=k$, and the above formula makes sense if we let $v_{n}=0$. Thus, using property (2), $\bar{\psi}\left(r^{\prime}\right)-\bar{\psi}(0)=r^{\prime}+v_{d^{\prime}}=r^{\prime}+\delta_{1}>s^{\prime} \omega>r^{\prime}$. Using the other half of (2), $\bar{\psi}(r+p)-\bar{\psi}(p)=r+p+v_{p^{\prime}}-\left(p+v_{p}\right)=r-\delta_{1}<s \omega<r$. So using Lemma 2, we have $\left(r^{\prime} / s^{\prime}, r / s\right) \subseteq R B(\Sigma)$, and we have proved the claim.

Now we wish to choose the interval $\left(r^{\prime} / s^{\prime}, r / s\right)$ as large as possible subject to (1) and (2). The convergents of $\omega$ provide a good choice but condition (1) on $r$ and $r^{\prime}$ may not always hold, so we need to distinguish several cases. The three cases we consider are (a) $n \mid p_{j}$, (b) $n \mid p_{j+1}$ and (c) $n \nmid p_{j}$ and $n \nmid p_{j+1}$, where we have let $j=j(n)$. Note that using the notation from above the theorem we have $\delta(h, \omega, n)=\left|\Delta_{j-1}\right|$. We shall assume that $j$ is even, the case where $j$ is odd is similar.

In case (a), since $p_{j}$ and $p_{j+1}$ are relatively prime, we have the (1) is satisfied if we let $r=p_{j+1}, s=q_{j+1}, r^{\prime}=p_{j+1}+p_{j}$ and $s^{\prime}=q_{j+1}+q_{j}$. Further, $\left(q_{j+1}+q_{j}\right) \omega-$ $\left(p_{j+1}+p_{j}\right)=\Delta_{j+1}+\Delta_{j}$ and $0<\Delta_{j+1}+\Delta_{j}<\left|\Delta_{j-1}\right|=\delta$, since $j$ is even. Also, $0<p_{j+1}-q_{j+1} \omega=-\Delta_{j+1}<\left|\Delta_{j-1}\right|=\delta$, and so (2) is satisfied. In case (b), using the fact that $n \backslash p_{j}$ and $n \backslash p_{j+2}$, one may choose $r^{\prime}=p_{j}, s^{\prime}=q_{j}, r=p_{j+1}+p_{j+2}$, and $s=q_{j+1}+q_{j+2}$ and use a similar argument to show that (1) and (2) hold for these choices. In case (c), let $r^{\prime}=p_{j}, s^{\prime}=q_{j}, r=p_{j+1}$ and $s=q_{j+1}$ and show that (1) and (2) hold. To complete the proof of the theorem, note that

$$
\frac{p_{j}}{q_{j}}<\frac{p_{j+1}+p_{j}}{q_{j+1}+q_{j}}<\omega<\frac{p_{j+1}+p_{j+2}}{q_{j+1}+q_{j+2}}<\frac{p_{j+1}}{q_{j+1}} .
$$

As is clear from the various cases considered at the end of this proof, one may obtain a better lower bound than the one given in the statement of the theorem in specific instances. For example, in case (c), there will be not invariant circles in the wider interval $\left\langle p_{j(n)} / q_{j(n)}, p_{j(n)+1} / q_{j(n)+1}\right\rangle$. Also, if a given $n$ falls into cases (a) or (b), it may be possible to increase $n$ so that it falls into case (c) while not increasing $j(n)$.

We also note that it may be possible to improve the general estimate in the theorem as follows. First, find a $\Sigma$ as in the first paragraph of the proof but with $\delta_{1}=\delta$. This would necessitate extending Mather's theorem slightly so that the image of $\Psi$ is the closure of $B_{\delta, n}$. This may be accomplished using the appropriate limit arguments. Then one would like to strengthen Lemma 2 so that its conclusion holds when $\bar{\psi}\left(r_{2}^{\prime}\right)-\bar{\psi}\left(r_{1}^{\prime}\right)=s^{\prime} \omega$ and $\bar{\psi}\left(r_{2}\right)-\bar{\psi}\left(r_{1}\right)=s \omega$. Using this, the proof of Theorem 3 would yield the same conclusion as before but with $j(n)-1$ in place of $j(n)$. Unfortunately, the extension of Lemma 2 is not possible without strengthening its hypothesis to include more information on how the measure on $\Sigma^{\prime}$ is distributed. The problem is that $\psi^{-1}(\bar{\psi}(b))$ for $b=r_{i}$ or $r_{i}^{\prime}$ can be two points, i.e. Denjoy minimal set can have a gap containing the vertical segment $\left\{r_{i}\right\} \times[0,1]$. This is in fact always the case for the minimal sets in Mather's theorem (see [M4], Theorem 15). This situation could perhaps be remedied by a closer analysis of how the function $\bar{\psi}$ varies with $\xi$ for a fixed $\Sigma^{\prime}$. 
As a last remark, we do some calculations with an example to illustrate the theorem. Let $f: \mathbf{R}^{2} \rightarrow \mathbf{R}^{2}$ be the much studied standard map

$$
(x, y) \mapsto\left(x+y-\frac{k}{2 \pi} \sin 2 \pi x, y-\frac{k}{2 \pi} \sin 2 \pi x\right) .
$$

In this case the generating function is $h\left(x, x^{\prime}\right)=\left(x^{\prime}-x\right)^{2} / 2+\left(k / 4 \pi^{2}\right) \cos 2 \pi x$.

Let us restrict to $n=2,0<\omega<1$ and $k<4 / 3$, since it is known that $f$ has no invariant circles when $k>4 / 3$ ([M2]). We also assume that $P(\omega)<1$ as this is reasonable in this range (see numerical calculations in $[\mathrm{M}-\mathrm{M}-\mathrm{P}]$ ). We thus have $C=6+k / 2 \pi$, and so

$$
N<\frac{4 C}{P(\omega)}+1<\frac{26}{P(\omega)} .
$$

Using the standard identities involving $p_{i}, q_{i}$ given before the theorem and letting $j=j(2)$, the width of the interval from which $\rho(\Gamma)$ is excluded is

$$
\frac{a_{j+2}}{\left(q_{j}+q_{j+1}\right)\left(q_{j+1}+q_{j+2}\right)} \stackrel{\text { def }}{=} D
$$

Now by definition, $q_{j-2}<N$ and $q_{j-1} \leqq N$. If we assume that $\omega$ is an irrational of constant type, i.e. there exists a constant $A$ with $a_{i} \leqq A$ for each partial quotient $a_{i}$ in $\left[0, a_{1}, a_{2}, \ldots\right]=\omega$, then using the identity $q_{j}=a_{j} q_{j-1}+q_{j-2}$, one has

$$
D>\frac{a_{j+2}}{\left(A^{5}+4 A^{4}+9 A^{3}+12 A^{2}+10 A+4\right) N^{2}} .
$$

Since $A \geqq 1$, calculus yields that the polynomial in the denominator is always less than $40 A^{5}$.

Combining the above using $a_{j+2} \geqq 1$, we get

$$
D>\frac{1}{40 A^{5}} \frac{(P(\omega))^{2}}{(26)^{2}}=\frac{1}{27040} \frac{(P(\omega))^{2}}{A^{5}} .
$$

Although we have made no attempt to optimize the estimate in this calculation, it does give some idea of the importance of the size of the partial quotients, $a_{i}$, of $\omega$. In particular, for a fixed value of $P(\omega)$, the irrational that "sweeps out of widest zone of instability" corresponds to $A=1$, i.e. the golden mean. This gives at least a vague notion of why this circle should be hardest to break.

Of much greater practical importance would be a computation of this type that was valid at rationals $p / q$. There is an analog of Theorem 3 for rationals (see [B-H], Theorem 5) but it assumes the existence of a non-monotone periodic orbit. What is lacking is a computable quantity that implies the existence of a non-monotone $p / q$-periodic orbit. 


\section{References}

[A-LD-A] Aubry, S., Le Daeron, P. Y., André, G.: Classical ground-states of a one-dimensional model for incommensurate structures, preprint

[B1] Birkhoff, G. D.: Surface transformations and their dynamical applications. Acta Mathematicae, 43, 44-47. (1920) Reprinted In: Collected Mathematical Papers of G. D. Birkhoff, Vol. II. New York: Dover 1968 pp. 195-202

[B2] Birkhoff, G. D.: Dynamical systems AMS Collegium, Vol. IX. Providence, R.I.: Am Math. Soc., 1927

[B3] Birkhoff, G. D.: Sur quelques courbes fermées remarquables Bull. S.M.F. 60, (1932), reprinted in Collected Math. Papers, Vol. II, pp. 418-443

[B4] Birkhoff, G. D., Sur l'existence de régions d'instabilité en dynamique. Ann Inst. Henri Poincaré 2, (1932), reprinted in Collected Math. Papers, Vol. II, pp. 444-461

[B-H] Boyland, P., Hall, G. R.: Invariant circles and the order structure of periodic orbits in monotone twist maps, preprint

[Fa] Fathi, A.: Une interprétation plus topologique de la démonstration du théorème de Birkhoff. appendix in [H2]

[Fu] Furstenberg, H.: Strict ergodicity and transformations of the torus. Am. J. Math. 83, 573-601, (1961)

[H-W] Hardy, G., Wright, E.: An introduction to the theory of numbers. Oxford: Oxford University Press, Fifth Edition, 1979

[H1] Herman, M.: Sur la conjugaison différentiable des difféomorphismes du cercle à des rotations. Publ. I.H.E.S. 49, 1979

[H2] Herman, M.: Sur les courbes invariantes par les difféomorphismes des l'anneau. Asterisque, 103-104, (1983)

[K] Katok, A.: Some remarks on the Birkhoff and Mather twist theorems. Ergodic Theory Dynam. Syst. 2, 183-194, (1982)

[M-M-P] Mackay, R., Meiss, J., Percival, I.: Transport in Hamiltonian systems. Physica 13D 55-81, (1984)

[M-P] Mackay, R., Percival, I.: Converse KAM: Theory and practice, preprint

[M-S] Mackay, R., Stark, J.: Lectures on orbits of minimal action for area-preserving maps, preprint

[M1] Mather, J.: Glancing billiards. Ergodic Theory Dynam. Syst. 2, 397-403, (1982)

[M2] Mather, J.: Non-existence of invariant circles. Ergodic Theory Dynam. Syst. 4, 301-309 (1984)

[M3] Mather, J.: A criterion for the non-existence of invariant circles, preprint

[M4] Mather, J.: More Denjoy minimal sets for area preserving diffeomorphisms, preprint

[N] Nitecki, Z.: Differentiable dynamics. Cambridge, MA: MIT Press 1971

[N-Z] Niven, I., Zuckerman, H.: An introduction to the theory of numbers, 2nd edition. New York: John Wiley 1966

Communicated by J. N. Mather

Received August 29, 1986; in revised form May 11, 1987 
